\title{
Increased Plasma Osmolar Gap Is Predictive of Contrast-Induced Acute Kidney Injury
}

\author{
Sejoong Kim,,${ }^{1,2}$ Jiyoon Sung, ${ }^{3}$ Woong Chul Kang, ${ }^{3}$ Shin Young Ahn, ${ }^{1,2}$ \\ Dong Ki Kim, ${ }^{2,4}$ Ho Jun Chin, ${ }^{1,2}$ Ki Young Na, ${ }^{1,2}$ Kwon Wook Joo, ${ }^{2,4}$ \\ Dong-Wan Chae ${ }^{1,2}$ and Jin Suk Han ${ }^{2,4}$ \\ ${ }^{1}$ Department of Internal Medicine, Seoul National University Bundang Hospital, Seongnam, Korea \\ ${ }^{2}$ Department of Internal Medicine, Seoul National University College of Medicine, Seoul, Korea \\ ${ }^{3}$ Department of Internal Medicine, Gachon University Gil Hospital, Incheon, Korea \\ ${ }^{4}$ Department of Internal Medicine, Seoul National University Hospital, Seoul, Korea
}

\begin{abstract}
Contrast-induced acute kidney injury (CIAKI) is a common complication after percutaneous coronary artery intervention $(\mathrm{PCl})$. It is urgent to find a novel, easily measurable and accurate predictor for the early detection of CIAKI. Hyperosmolarity and large amounts of contrast media are risk factors for CIAKI. However, there is no study on plasma osmolar gap as a predictor of CIAKI. We enrolled 89 patients undergoing elective $\mathrm{PCl}$ and tested changes of serum sodium, osmolar gap, and renal function at $0,6,12$ and 24 hours. Plasma osmolar gap was calculated using the following formula: measured plasma osmolarity - [2(Na) + serum urea nitrogen/2.8 + glucose/18]. CIAKI was defined as follows: increase in serum creatinine of $\geq 50 \%$, increase in serum creatinine of $\geq 0.3 \mathrm{mg} / \mathrm{dL}$, or decrease in estimated glomerular filtration rate of $\geq 25 \%$ within 24 hours after PCI. The incidence of CIAKI was $13.5 \%(12 / 89$ patients). The CIAKI group had higher plasma osmolar gaps 6 hours after PCI. The adjusted hazard ratio of the plasma osmolar gap from hour $6(1-\mathrm{mOsm} / \mathrm{L}$ increments) to the development of CIAKI was $1.12(95 \%$ confidence interval $[\mathrm{Cl}], 1.01-1.26 ; P=0.041)$. Sensitivity and specificity of $7 \mathrm{mOsm} / \mathrm{L}$ or higher plasma osmolar gap at hour 6 were $70.0 \%$ and $76.6 \%$, respectively (area under the ROC curve $=0.77$ [95\% Cl, 0.65-0.89]). Increased plasma osmolar gap may precede the development of CIAKI in patients undergoing $\mathrm{PCl}$. In conclusion, plasma osmolar gap may be a useful predictor for the development of CIAKI.
\end{abstract}

Keywords: acute kidney injury; biomarker; contrast media; coronary angiography; osmolality Tohoku J. Exp. Med., 2012 Oct, 228 (2), 109-117. C 2012 Tohoku University Medical Press

As the variety of diagnostic and interventional studies using contrast media has grown rapidly, the risk of contrastinduced acute kidney injury (CIAKI) has also increased. The number of patients at the risk for developing CIAKI, including those of advanced age with diabetes and chronic kidney disease, is increasing, although some preventive methods have been performed (Kim et al. 2010). CIAKI can prolong hospital stay and lead to early and late worse outcomes (McCullough 2008). Some studies have been performed to determine predictors or biomarkers for the early detection of CIAKI, including serum cystatin C (Briguori et al. 2010) and neutrophil gelatinase-associated lipocalin (Bachorzewska-Gajewska et al. 2006; Hirsch et al. 2007). However, measurements of these parameters may not be available in practice. Thus, we need to find a new, easily measurable, inexpensive and accurate predictor for the early detection of CIAKI.

The osmolarity of contrast media can be directly cyto- toxic and impair cell volume regulation, and increased plasma viscosity can impair renal blood flow, which may lead to CIAKI (Galtung et al. 2000; Persson et al. 2005). A potential candidate biomarker to measure the osmotic effects of contrast media on renal tubular injury is plasma osmolar gap. A previous study showed that there was no association among contrast agents, plasma osmolarity and osmolar gap 30 minutes after the performance of a contrastenhanced computed tomography; however, that study only enrolled 14 children. In contrast, increased plasma osmolar gap plus hyponatremia may reflect organ dysfunction in critically ill patients (Guglielminotti et al. 2002). However, there is no study on the diagnostic usefulness of plasma osmolar gap for the early detection of CIAKI. We evaluated whether plasma osmolar gap could predict CIAKI in patients undergoing elective percutaneous coronary artery interventions (PCI).

Received July 26, 2012; accepted August 16, 2012. Published online September 5, 2012; doi: 10.1620/tjem.228.109.

Correspondence: Jin Suk Han, M.D., Ph.D., Department of Internal Medicine, Seoul National University Hospital, 28 Yongon-dong,

Chongno-gu, Seoul 110-744, Korea.

e-mail: jshan@snu.ac.kr 


\section{Methods}

\section{Study participants}

We enrolled 89 consecutive patients undergoing elective PCI for stable angina at the Gachon University Gil Hospital. During the procedure, cardiologists decided to perform either coronary angiography alone or angioplasty with stenting. We excluded patients with preexisting chronic kidney disease (estimated glomerular filtration rate $\left[\right.$ eGFR] $<60 \mathrm{~mL} / \mathrm{min} / 1.73 \mathrm{~m}^{2}$ ), hypersensitivity to contrast media, liver cirrhosis, syndrome of inappropriate anti-diuretic hormone, hypothyroidism or those who had received mannitol or intravenous immunoglobulin therapy. Informed consent was obtained from all study participants, and the study was approved by the institutional review boards of Gachon University Gil Hospital.

None of the study patients had received nephrotoxic drugs within at least 1 week preceding or during the study period. Volume loading with intravenous normal saline $(1.0-1.5 \mathrm{~mL} / \mathrm{kg} / \mathrm{h})$ was started 2-4 hours before the procedure and continued for 6 hours afterwards. Intravenous diuretics and nephrotoxic drugs, such as metformin, were withdrawn 72 hours before the procedure. All patients received low osmolar contrast media (iopromide, Ultravist ${ }^{\mathbb{R}}$; Bayer Schering Pharma, Berlin, Germany).

\section{Study protocol}

Baseline demographic data and data regarding patient history of hypertension, hyperlipidemia, diabetes mellitus, and smoking were collected. Hypertension was defined as a self-reported history of hypertension and/or use of antihypertensive medications or blood pressure $\geq 140 / 90 \mathrm{mmHg}$. Diabetes was defined as a self-reported history of diabetes and/or use of anti-diabetic treatment or a fasting plasma glucose $\geq 126 \mathrm{mg} / \mathrm{dl}$. Cardiovascular disease was defined as a history of congestive heart failure and cerebrovascular accidents. Smoking was defined as any cigarette smoking within one year of PCI. Height, body weight, and blood pressure were measured in all patients. Serum levels of creatinine, electrolytes, blood urea nitrogen and glucose, plasma osmolarity, and urinary levels of electrolytes, creatinine, and osmolarity were evaluated before and 6,12, and $24 \mathrm{~h}$ after the PCI. Serum cystatin C levels were assessed before and $24 \mathrm{~h}$ after the procedure. Creatinine was measured using the rate-blanked and compensated Jaffé method, and the automated systems were calibrated (Hitachi High-Tech, Tokyo, Japan). Serum cystatin C was measured using HiSens CystatinC LTIA kit (HBI Co., Anyang, Korea) on a Toshiba chemical analyzer (Toshiba, Nasushiobara, Japan). Plasma osmolarity was measured with the Fiske One-Ten freeze-point osmometer (Radiometer, Copenhagen, Denmark).

\section{Definition}

CIAKI was diagnosed if one of the following criteria were satisfied: increase in serum creatinine of $50 \%$ or more, increase in serum creatinine of $0.3 \mathrm{mg} / \mathrm{dL}$ or more, or decrease in eGFR of $25 \%$ or more within 24 hours after PCI. eGFR was calculated using the formula developed and validated in the Modification of Diet in Renal Disease (Levey et al. 1999), and the Korean coefficients for these equations were applied (Lee et al. 2010). Calculated osmolarity was estimated using the formula $2(\mathrm{Na}[\mathrm{mmol} / \mathrm{L}])+$ serum urea nitrogen $[\mathrm{mg} / \mathrm{dL}] / 2.8$ + glucose $[\mathrm{mg} / \mathrm{dL}] / 18$, and plasma osmolar gap was defined as the difference between measured osmolarity and calculated osmolarity (Worthley et al. 1987).

\section{Statistical analysis}

Before beginning the study, the sample size was estimated assuming a development of CIAKI in $15 \%$ of patients, and the difference of osmolar gaps in $4.0 \pm 5.0$ (mean \pm s.D.) $\mathrm{mOsm} / \mathrm{L}$. A sample size of 100 patients would be required to detect a statistically significant difference with a power of $80 \%(\alpha=0.05)$ Power analysis of two-sample $t$-tests was completed using PASS 2008 (NCSS, Kaysville, UT, USA).

Data were reported as mean \pm S.D. or as number (percentage). Differences between the two groups at each time point were tested with an unpaired Student's $t$-test, and changes of variables in each group were tested with a paired $t$-test. Differences of categorical variables between the two groups were tested with a chi-square test and with the Fisher's exact test when the variables had an expected $n<5$. Repeated measures analysis of variance was used to evaluate changes in eGFR, osmolar gap, and serum sodium as the repeated measure, and the CIAKI group was the between-group factor. Logistic regression analysis was performed for the assessment of an independent association of the plasma osmolar gap with CIAKI. Diagnostic performance was expressed in terms of sensitivity, specificity, positive and negative predictive values and as the area under receiver operating characteristics (ROC) curves. Data analysis was conducted using SPSS 18.0 for Windows (SPSS Inc., Chicago, IL, USA). A $P<0.05$ was considered to be statistically significant.

\section{Results}

The incidence of CIAKI was $13.5 \%$ (12/89 patients). As shown in Table 1, the CIAKI group has lower body weight, but this difference was not statistically significant. The amount of contrast media received was more likely to be higher in the CIAKI group, and the proportion of interventional procedures was higher in the CIAKI group. There were no differences in comorbidity or medications between the two groups.

At baseline, there were no differences in eGFR, serum creatinine, or serum cystatin $\mathrm{C}$ between the two groups (Table 2). eGFR at hour 6 in the CIAKI group was significantly lower than than in the no-CIAKI group. Furthermore, eGFR in the CIAKI group gradually decreased until 24 hours after PCI. In the no-CIAKI group, eGFR was slightly increased at hour 6 and 12; there was no difference of eGFR between baseline and hour 24 (Fig. 1A). Serum creatinine at hour 6 and serum cystatin $C$ at hour 24 in the CIAKI group were higher than in the control group. Serum creatinine levels at hour 12 and 24 in the CIAKI group were also higher, but not significant.

Plasma osmolar gap was increased 6 hours and 12 hours after the procedure in the CIAKI group, while it decreased to the same level as the control group 24 hours after PCI (Fig. 1B). There was no difference in serum sodium at baseline between the two groups. In contrast, serum sodium levels at hour 6,12 , and 24 in the CIAKI group were lower than those in the control group (Fig. 1C).

In the logistic regression analysis, the odds ratio of the plasma osmolar gap at hour $6(1 \mathrm{mOsm} / \mathrm{L}$ increment $)$ for predicting CIAKI was 1.12 (95\% confidence interval [CI], 1.03-1.22). After the adjustment of covariates, including 
Table 1. Baseline characteristics.

\begin{tabular}{|c|c|c|c|}
\hline & No CIAKI $(n=77)$ & CIAKI $(n=12)$ & $P$ \\
\hline Male (\%) & $48(62.3)$ & $6(50.0)$ & 0.416 \\
\hline Age (year) & $61.4 \pm 11.8$ & $64.7 \pm 14.3$ & 0.384 \\
\hline Height (cm) & $161 \pm 8.87$ & $162 \pm 7.55$ & 0.868 \\
\hline Body weight_pre (kg) & $66.4 \pm 11.7$ & $60.3 \pm 12.5$ & 0.098 \\
\hline $\mathrm{eGFR}\left(\mathrm{mL} / \mathrm{min} / 1.73 \mathrm{~m}^{2}\right)$ & $106 \pm 21.7$ & $110 \pm 42.7$ & 0.722 \\
\hline \multicolumn{4}{|l|}{ Comorbidity } \\
\hline Diabetes $(\%)$ & $18(23.4)$ & $4(33.3)$ & 0.481 \\
\hline Hypertension (\%) & $37(48.1)$ & $6(50.0)$ & 0.900 \\
\hline Cardiovascular disease $(\%)$ & $18(23.4)$ & $5(41.7)$ & 0.178 \\
\hline Current Smoker & $16(20.8)$ & $5(41.7)$ & 0.238 \\
\hline \multicolumn{4}{|l|}{ Medication history } \\
\hline ACEi/ARB (\%) & $22(28.6)$ & $4(33.3)$ & 0.741 \\
\hline Beta-blocker $(\%)$ & $12(14.5)$ & $4(33.3)$ & 0.115 \\
\hline Calcium channel blocker (\%) & $19(24.7)$ & $3(25.0)$ & 1.000 \\
\hline Diuretics (\%) & $7(9.1)$ & $2(16.7)$ & 0.764 \\
\hline Volume of isotonic saline $(\mathrm{mL})$ & $997 \pm 257$ & $1,015 \pm 367$ & 0.901 \\
\hline Angioplasty (\%) & $25(32.5)$ & $8(66.7)$ & 0.050 \\
\hline Contrast media (mL) & $162 \pm 87.4$ & $258 \pm 137$ & 0.037 \\
\hline
\end{tabular}

eGFR, estimated glomerular filtration rate; ACEi/ARB, angiotensin converting enzyme inhibitor/angiotensin receptor blocker.

age, gender, diabetes, baseline serum sodium level, contrast media and angioplasty, the adjusted odds ratio of the plasma osmolar gap at hour $6(1 \mathrm{mOsm} / \mathrm{L}$ increment $)$ to the development of CIAKI was 1.12 (95\% CI, 1.01-1.26) (Table 3). There were no linear correlations between volume of contrast media and plasma osmolar gaps at hour 6 in both noCIAKI group and CIAKI group (Fig. 2).

The area under ROC curve was 0.772 (95\% CI, 0.634$0.909)$ for plasma osmolal gap at hour $6,0.734(95 \% \mathrm{CI}$, 0.603-0.866) for cystatin C increase, 0.696 (95\% CI, 0.4780.914 ) for serum cystatin $\mathrm{C}$ at hour 24 (Fig. 3). Thus, the value of the plasma osmolar gap at hour 6 was not inferior to that of each cystatin $\mathrm{C}$ parameters. The sensitivity and specificity of a plasma osmolar gap $>7 \mathrm{mOsm} / \mathrm{L}$ at hour 6 were above $70.0 \%$ (Table 4 ). The sensitivity and specificity of cystatin $\mathrm{C}$ increase $>-1 \%$ and cystatin $\mathrm{C}$ at hour $24>1.1$ $\mathrm{mg} / \mathrm{dL}$ were approximately $70.0 \%$.

\section{Discussion}

We found that an increased osmolar gap, which is related to contrast media and hyponatremia, may precede the development of CIAKI in patients undergoing PCI, and that an osmolar gap at hour 6 may be a useful predictor of CIAKI. Regarding the pathophysiology of CIAKI, the plasma osmolar gap can reflect the osmotic effects of contrast media. In addition, the measurement of the plasma osmolar gap is easily assessable and inexpensive; therefore, physicians can immediately apply this measurement to their practice.

In a normally functioning kidney, contrast media is almost completely excreted within 8 hours (Lawaczeck et al. 2011). One study demonstrated that contrast media does not increase plasma osmolarity or produce an osmolar gap between the time 0 and 30 minutes after contrast administration (George and Shannon 2009); their study showed no data on changes in renal function. In our study, there was no change in plasma osmolarity or plasma osmolar gap in the no-CIAKI group, which is consistent with a previous report (George and Shannon 2009). In contrast, the plasma osmolar gap at hour 6 was significantly increased in patients with CIAKI. Contrast media induces renal cell apoptosis in a dose-dependent manner (Romano et al. 2008). These changes can occur within a few hours and may lead to kidney injury (Galtung et al. 2000; Bachorzewska-Gajewska et al. 2006; Romano et al. 2008). Increased plasma osmolar gap at hour 6 may reflect kidney damage related to contrast media. In addition, a high plasma osmolar gap at hour 6 can be the consequence of delayed renal excretion of contrast media due to decreased renal function which could increase the risk of CIAKI because these patients have a higher chance of exposure to contrast media.

According to our data, changes in measured plasma osmolarity were minimal in the CIAKI group. An increased osmolar gap at hour 6 may be due to a decrease in serum sodium at hour 6 , which leads to a decreased calculated osmolarity. Symptomatic severe hyponatremia develops infrequently after elective cardiac procedures (Boulos et al. 2001), but asymptomatic mild hyponatremia commonly occurs after PCI. There are several explanations for the development of hyponatremia related to procedure requir- 


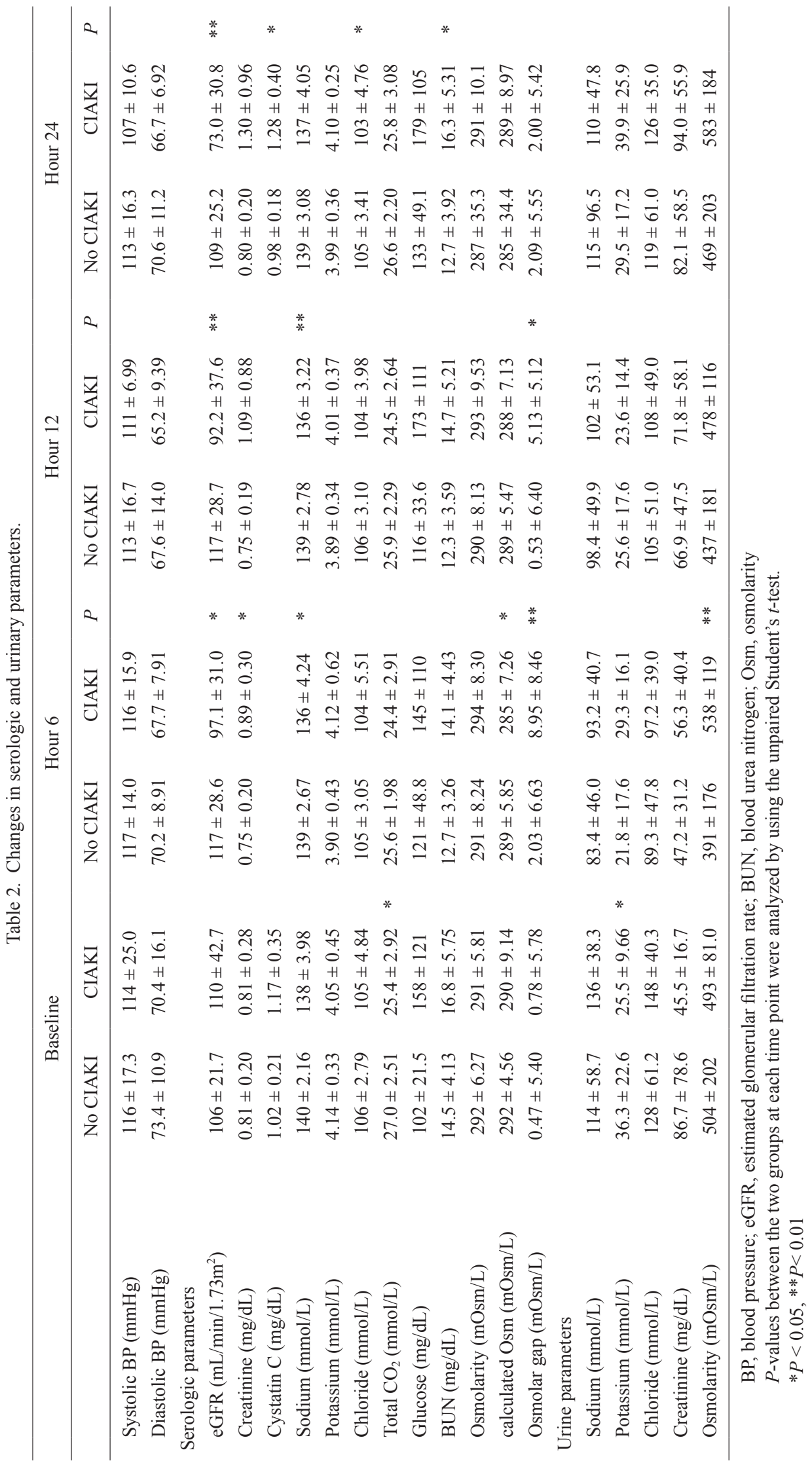


A

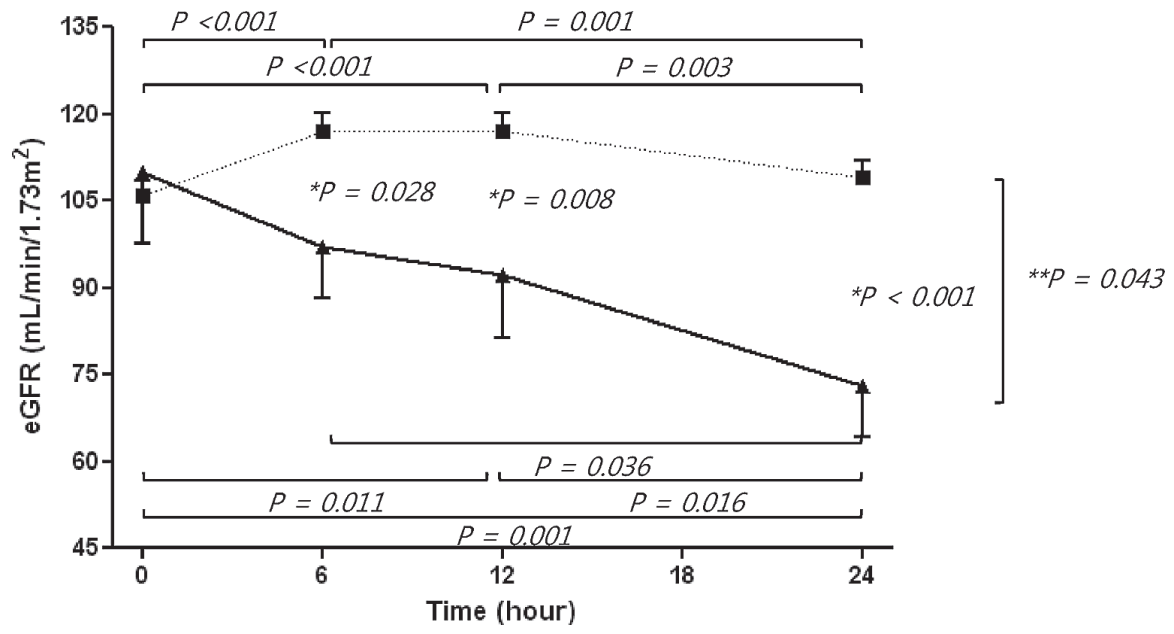

B

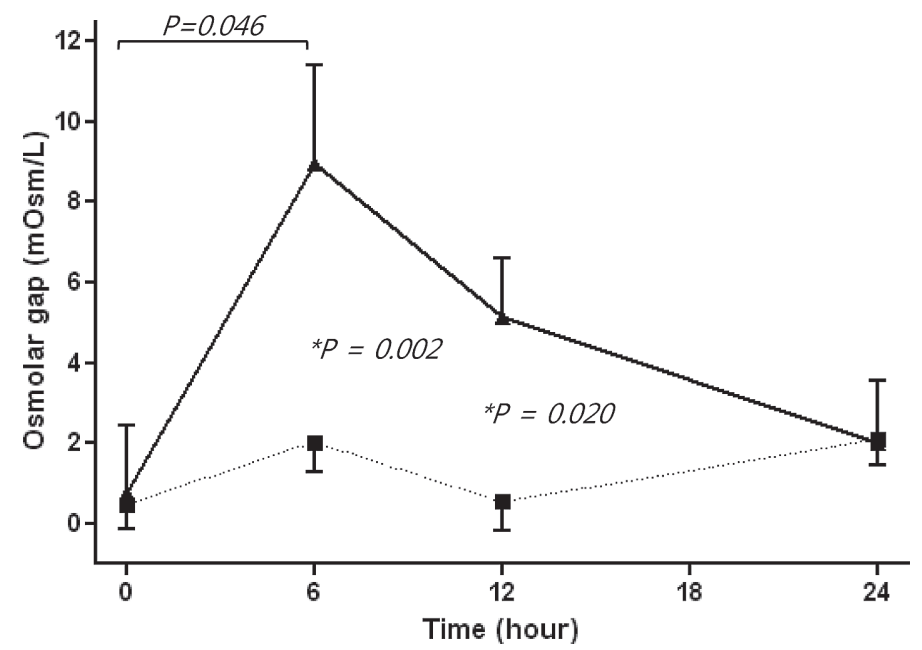

$\mathrm{C}$

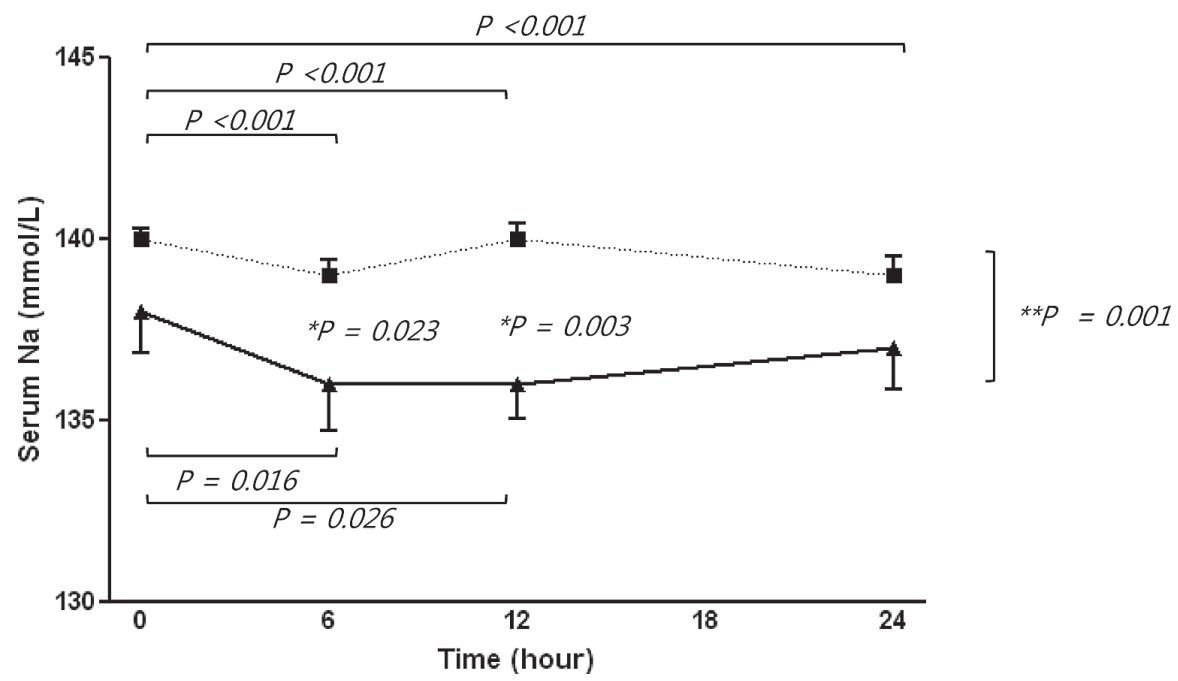

Fig. 1. Change of estimated glomerular filtration rate (eGFR) (A), plasma osmolar gap (B), and serum sodium levels (C) between the contrast-induced acute kidney injury (CIAKI) group (solid lines, $n=77$ ) and no-CIAKI group (dotted line, $n=12)$.

Data were expressed as mean \pm S.D. $P$-values were analyzed by using the paired $t$-test within the group, $* P$-values were analyzed by using the unpaired Student's $t$-test between the two groups at each time point, and $* * P$-values were analysed by using repeated measures analysis of variance. 
Table 3. Logistic regression analysis of plasma osmolar gap for predicting contrast-induced acute kidney injury.

\begin{tabular}{lcccc}
\hline \multicolumn{1}{c}{ Parameters } & \multicolumn{2}{c}{ Univariable } & \multicolumn{2}{c}{ Multivariable } \\
\cline { 2 - 5 } & $P$ & OR (95\% CI) & $P$ & OR (95\% CI) \\
\hline Age (1-year increment) & 0.380 & $1.023(0.972-1.078)$ & 0.624 & $0.984(0.921-1.050)$ \\
Male (vs. female) & 0.419 & $1.655(0.488-5.617)$ & 0.587 & $1.581(0.303-8.247)$ \\
Diabetes (yes vs. no) & 0.460 & $1.639(0.442-6.081)$ & 0.754 & $0.754(0.129-4.423)$ \\
Serum sodium (1-mmol/L increment) & 0.011 & $0.735(0.580-0.931)$ & 0.023 & $0.661(0.463-0.944)$ \\
Plasma osmolar gap at hour 6 (1-mOsm/L increment) & 0.007 & $1.123(1.032-1.220)$ & 0.041 & $1.123(1.005-1.255)$ \\
Contrast media (> 100 mL vs. $<100 \mathrm{~mL})$ & 0.023 & $4.966(1.242-19.84)$ & 0.108 & $6.471(0.665-62.92)$ \\
Angioplasty (vs. angiography) & 0.031 & $4.160(1.143-15.13)$ & 0.690 & $0.656(0.083-5.191)$ \\
\hline
\end{tabular}

$\mathrm{OR}$, odds ratio; $\mathrm{CI}$, confidence interval

A

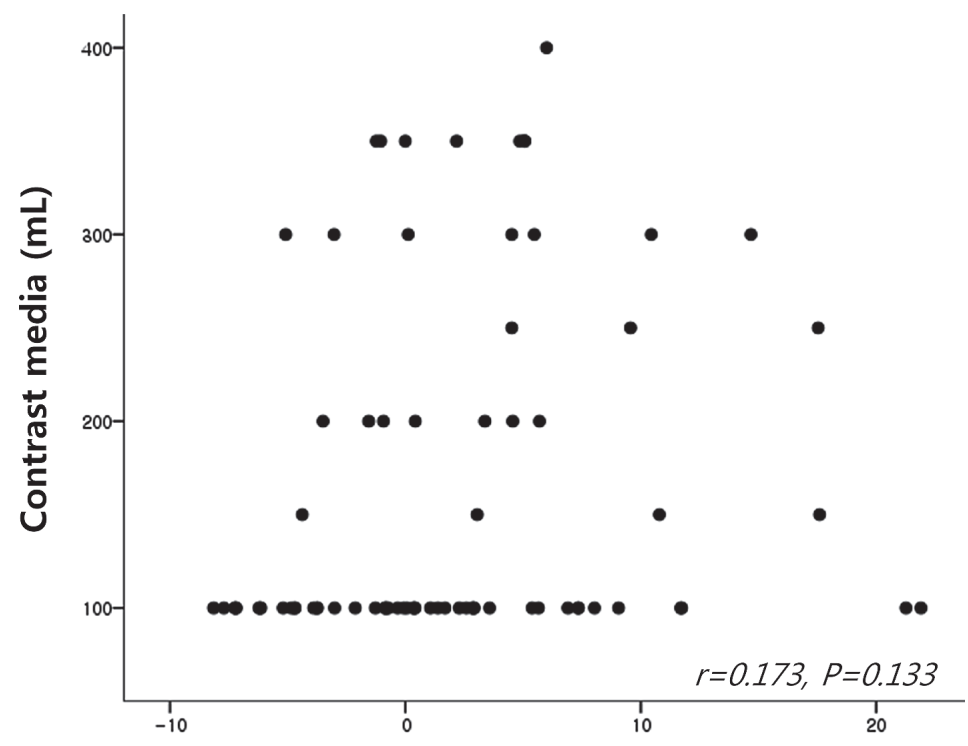

Osmolar gap at hour $6(\mathrm{mmol} / \mathrm{L})$

B

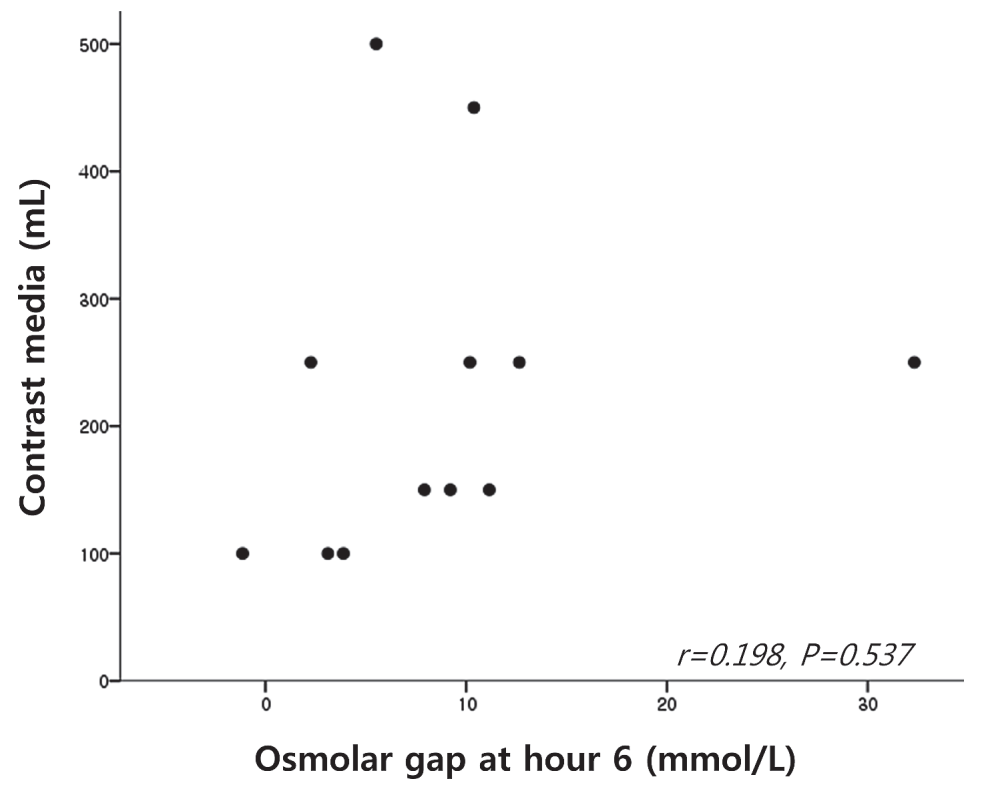

Fig. 2. Correlation between volume of contrast media and osmolar gap at hour 6 .

There were no linear correlations in no-CIAKI group (A, $n=77)$ and CIAKI group (B, $n=12)$. 


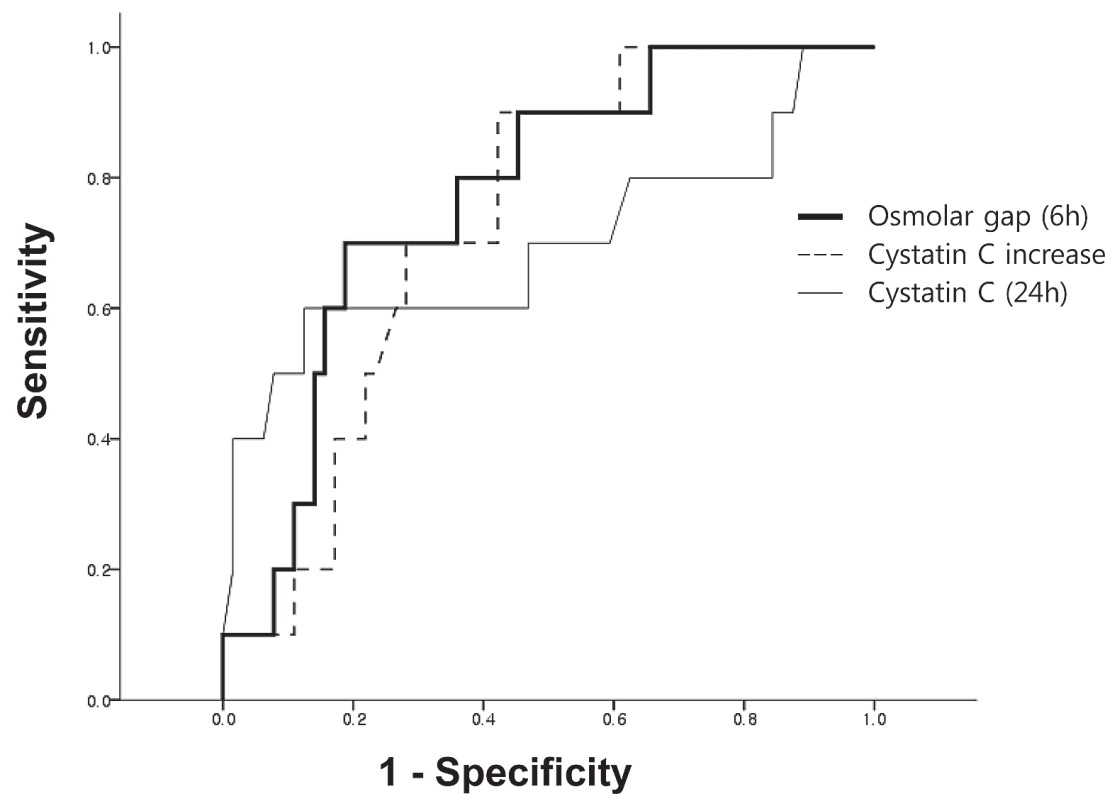

Fig. 3. Comparison of receiver operating characteristics curves for four serum parameters to predict development of contrast-induced acute kidney injury.

The area under the ROC curve is as follows: plasma osmolar gap at hour 6, 0.772 (95\% confidence interval [CI], 0.6340.909); cystatin C increase between hour 0 and hour 24, 0.734 (95\% CI, 0.603-0.866); serum cystatin C at hour 24, 0.696 (95\% CI, 0.478-0.914).

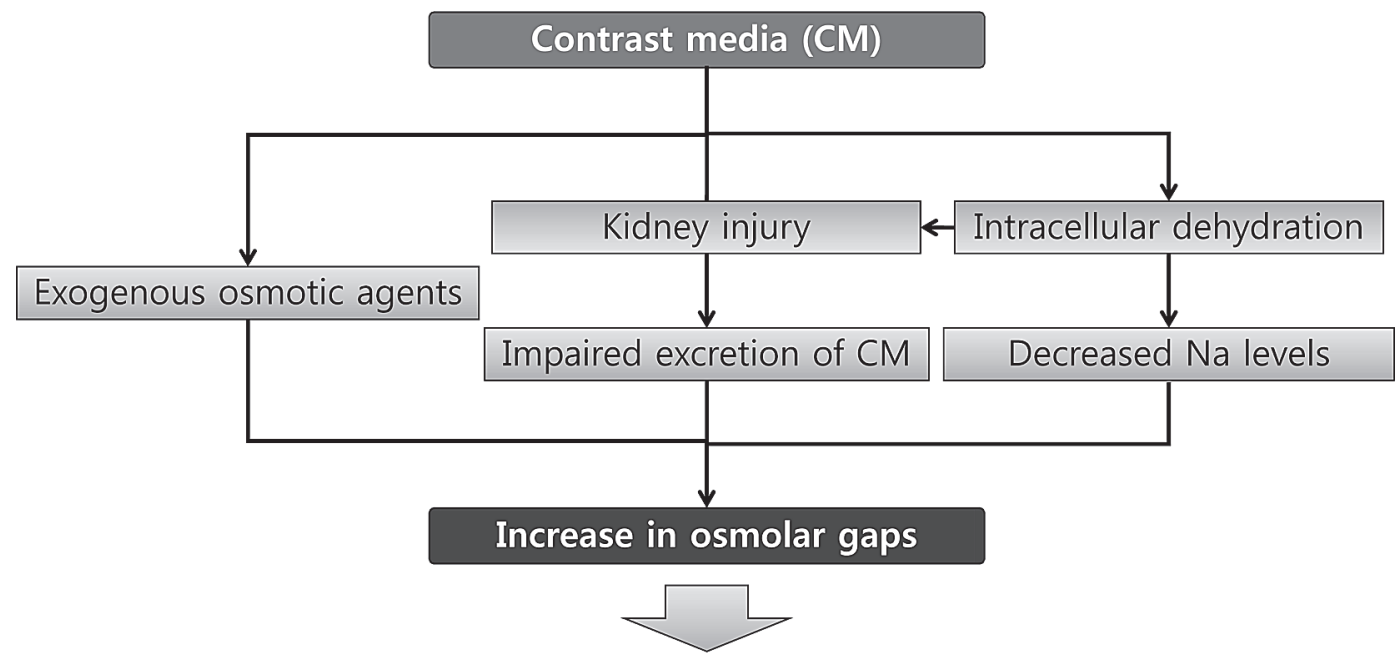

\section{Contrast induced acute kidney injury}

Fig. 4. Suggested mechanisms of increased osmolar gap.

ing contrast media. A potential mechanism for hyponatremia could be contrast-induced translocational hyponatremia (Sirken et al. 2004). The osmolar effects of contrast media may result in osmotic water movement out of cells, thereby decreasing sodium level by endogenous dilution, producing a so-called translational hyponatremia (Adrogue and Madias 2000; Sirken et al. 2004). Some experimental studies showed that contrast media can induce prolonged shrinkage of renal tubular cells and red blood cells and decrease these cells' ability to regulate cellular volume (Galtung et al. 2000, 2001, 2002), which may be an early precursor to apoptosis (Maeno et al. 2000). Therefore, transient contrast-induced translocational hyponatremia may be a main mechanism of increased plasma osmolar gap at hour 6 in the CIAKI group of our study. It is possible that osmotic diuresis secondary to contrast medium may contribute to the development of hyponatremia since urine osmolarity at hour 6 was increased in the CIAKI group compared to the control group. The administration of hypotonic fluids appears to be one of the causes of an acute reduction in serum sodium levels, together with an impaired urinary dilutional capacity (Aronson et al. 2002). In our 
Table 4. Sensitivity and specificity of four plasma parameters at various cut-off points to predict contrast-induced acute kidney injury.

\begin{tabular}{cccc}
\hline Parameters & Cut-off point & Sensitivity (\%) & Specificity (\%) \\
\hline & 5.0 & 70.0 & 68.7 \\
Osmolar gap at hour 6 (mOsm/L) & 6.0 & 70.0 & 75.0 \\
& 7.0 & 70.0 & 76.6 \\
& 8.0 & 60.0 & 81.2 \\
cystatin C increase (\%) & -2 & 70.0 & 70.3 \\
& -1 & 70.0 & 73.4 \\
& 0 & 60.0 & 76.6 \\
cystatin C at hour 24 (mg/L) & 1 & 50.0 & 32.8 \\
& 0.9 & 80.0 & 56.2 \\
\hline
\end{tabular}

study, none of the enrolled patients received hypotonic saline.

In a logistic regression analysis used to confirm the dose-responsiveness relationship, the plasma osmolar gap at hour 6 was positively associated with the development of CIAKI. At baseline, patients in the CIAKI group received coronary angioplasty at a high frequency, including ballooning or stent insertion, and received greater volumes of contrast media compared to control patients. After adjusting for those factors in multiple logistic regression analysis, plasma osmolar gap at hour 6 was still significantly associated with an increased incidence of CIAKI. Several studies have demonstrated a positive correlation between contrast media volume and CIAKI (Manske et al. 1990; Taliercio et al. 1991; Rudnick et al. 1995), while other studies found no relationship (Moore et al. 1992; Durham et al. 2002; Mehta et al. 2002). In no-CIAKI group or CIAKI group, there was no correlation between osmolar gaps at hour 6 and contrast media volume (Fig. 2). Although contrast media volume was positively correlated with the development of CIAKI in univariable analysis of our data, there was no significance in correlation after the adjustment of covariables (Table 3). It suggests that osmolar gap at hour 6 may reflect kidney injury independently of contrast media volume.

Cystatin $\mathrm{C}$ is one of the most reliable markers for the early diagnosis of CIAKI (Briguori et al. 2010). Briguori et al. (2010) demonstrated that AUC charting cystatin C increase in a ROC curve analysis is over 0.9. In our study, the same parameter of cystatin $\mathrm{C}$ increase was calculated for the prediction of CIAKI, for which the AUC was 0.735 . Different sample sizes between the two studies may affect the AUC. The AUC of the plasma osmolar gap at hour 6 was slightly higher than that of cystatin $\mathrm{C}$ increase in our study. This finding suggests that the plasma osmolar gap at hour 6 may be a comparable marker to cystatin $\mathrm{C}$ increase for the early detection of CIAKI.
Fig. 4 shows suggested mechanisms of increased osmolar gaps after contrast study. Contrast media directly increases plasma osmolar gaps and indirectly impairs cellular volume regulation, which leads to hyponatremia and rising osmolar gaps. In addition, CIAKI can contribute to decrease in renal function and impaired excretion of contrast media. Therefore, increased osmolar gaps may reflect and predict the development of CIAKI.

We had some limitations in this study. Plasma osmolar gap might not be of value when using iso-osmolar agents. We observed the patients for only 24 hours, which might lead to misclassification of patients with delayed renal injury. There is a possibility that the role of increased osmolar gap may be the epiphenomenon. Further studies should be needed to evaluate direct or indirect role of this biomarker.

We found that an increased plasma osmolar gap may predict the development of CIAKI within 6 hours after cardiac intervention. Patients with an increased plasma osmolar gap after administration of contrast media may be candidates for further preventive strategies to protect against CIAKI.

\section{Acknowledgments}

This work was supported by Astellas Research Grants from the Korean Society of Nephrology (2011).

\section{Conflict of Interest}

The authors have no financial conflict of interest.

\section{References}

Adrogue, H.J. \& Madias, N.E. (2000) Hyponatremia. N. Engl. J. Med., 342, 1581-1589.

Aronson, D., Dragu, R.E., Nakhoul, F., Hir, J., Miller, A., Boulos, M., Zinder, O., Green, J., Mittleman, M.A. \& Markiewicz, W. (2002) Hyponatremia as a complication of cardiac catheterization: a prospective study. Am. J. Kidney Dis., 40, 940-946. 
Bachorzewska-Gajewska, H., Malyszko, J., Sitniewska, E., Malyszko, J.S. \& Dobrzycki, S. (2006) Neutrophil-gelatinaseassociated lipocalin and renal function after percutaneous coronary interventions. Am. J. Nephrol., 26, 287-292.

Boulos, M., Nakhoul, F., Hir, J. \& Markiewicz, W. (2001) Acute symptomatic hyponatremia complicating invasive cardiac procedures: a report of three patients. Catheter. Cardiovasc. Interv., 53, 542-545.

Briguori, C., Visconti, G., Rivera, N.V., Focaccio, A., Golia, B., Giannone, R., Castaldo, D., De Micco, F., Ricciardelli, B. \& Colombo, A. (2010) Cystatin c and contrast-induced acute kidney injury. Circulation, 121, 2117-2122.

Durham, J.D., Caputo, C., Dokko, J., Zaharakis, T., Pahlavan, M., Keltz, J., Dutka, P., Marzo, K., Maesaka, J.K. \& Fishbane, S. (2002) A randomized controlled trial of n-acetylcysteine to prevent contrast nephropathy in cardiac angiography. Kidney Int., 62, 2202-2207.

Galtung, H.K., Loken, M. \& Sakariassen, K.S. (2000) Effect of radiologic contrast material on cell volume regulation in proximal renal tubules from trout (salmo trutta). Acad. Radiol., 7, 911-919.

Galtung, H.K., Loken, M. \& Sakariassen, K.S. (2001) Effect of radiologic contrast media on cell volume regulation in rabbit proximal renal tubules. Acad. Radiol., 8, 398-404.

Galtung, H.K., Sorlundsengen, V., Sakariassen, K.S. \& Benestad, H.B. (2002) Effect of radiologic contrast media on cell volume regulatory mechanisms in human red blood cells. Acad. Radiol., 9, 878-885.

George, M. \& Shannon, M. (2009) Intravenous contrast agents and associated changes in serum osmolality. Pediatr. Emerg. Care, 25, 555-557.

Guglielminotti, J., Pernet, P., Maury, E., Alzieu, M., Vaubourdolle, M., Guidet, B. \& Offenstadt, G. (2002) Osmolar gap hyponatremia in critically ill patients: evidence for the sick cell syndrome? Crit. Care Med., 30, 1051-1055.

Hirsch, R., Dent, C., Pfriem, H., Allen, J., Beekman, R.H. 3rd., Ma, Q., Dastrala, S., Bennett, M., Mitsnefes, M. \& Devarajan, P. (2007) NGAL is an early predictive biomarker of contrastinduced nephropathy in children. Pediatr. Nephrol., 22, 20892095.

Kim, S.M., Cha, R.H., Lee, J.P., Kim, D.K., Oh, K.H., Joo, K.W., Lim, C.S., Kim, S. \& Kim, Y.S. (2010) Incidence and outcomes of contrast-induced nephropathy after computed tomography in patients with ckd: a quality improvement report. Am. J. Kidney Dis., 55, 1018-1025.

Lawaczeck, R., Jost, G. \& Pietsch, H. (2011) Pharmacokinetics of contrast media in humans: model with circulation, distribution, and renal excretion. Invest. Radiol., 46, 576-585.
Lee, C.S., Cha, R.H., Lim, Y.H., Kim, H., Song, K.H., Gu, N., Yu, K.S., Lim, C.S., Han, J.S., Kim, S. \& Kim, Y.S. (2010) Ethnic coefficients for glomerular filtration rate estimation by the modification of diet in renal disease study equations in the korean population. J. Korean Med. Sci., 25, 1616-1625.

Levey, A.S., Bosch, J.P., Lewis, J.B., Greene, T., Rogers, N. \& Roth, D. (1999) A more accurate method to estimate glomerular filtration rate from serum creatinine: a new prediction equation. Modification of diet in renal disease study group. Ann. Intern. Med., 130, 461-470.

Maeno, E., Ishizaki, Y., Kanaseki, T., Hazama, A. \& Okada, Y. (2000) Normotonic cell shrinkage because of disordered volume regulation is an early prerequisite to apoptosis. Proc. Natl. Acad. Sci. USA., 97, 9487-9492.

Manske, C.L., Sprafka, J.M., Strony, J.T. \& Wang, Y. (1990) Contrast nephropathy in azotemic diabetic patients undergoing coronary angiography. Am. J. Med., 89, 615-620.

McCullough, P.A. (2008) Contrast-induced acute kidney injury. $J$. Am. Coll. Cardiol., 51, 1419-1428.

Mehta, R.L., Pascual, M.T., Soroko, S. \& Chertow, G.M. (2002) Diuretics, mortality, and nonrecovery of renal function in acute renal failure. JAMA, 288, 2547-2553.

Moore, R.D., Steinberg, E.P., Powe, N.R., Brinker, J.A., Fishman, E.K., Graziano, S. \& Gopalan, R. (1992) Nephrotoxicity of high-osmolality versus low-osmolality contrast media: randomized clinical trial. Radiology, 182, 649-655.

Persson, P.B., Hansell, P. \& Liss, P. (2005) Pathophysiology of contrast medium-induced nephropathy. Kidney Int., 68, 14-22.

Romano, G., Briguori, C., Quintavalle, C., Zanca, C., Rivera, N.V., Colombo, A. \& Condorelli, G. (2008) Contrast agents and renal cell apoptosis. Eur. Heart J., 29, 2569-2576.

Rudnick, M.R., Goldfarb, S., Wexler, L., Ludbrook, P.A., Murphy, M.J., Halpern, E.F., Hill, J.A., Winniford, M., Cohen, M.B. \& VanFossen, D.B. (1995) Nephrotoxicity of ionic and nonionic contrast media in 1196 patients: a randomized trial. The iohexol cooperative study. Kidney Int., 47, 254-261.

Sirken, G., Raja, R., Garces, J., Bloom, E. \& Fumo, P. (2004) Contrast-induced translocational hyponatremia and hyperkalemia in advanced kidney disease. Am. J. Kidney Dis., 43, e31-35.

Taliercio, C.P., Vlietstra, R.E., Ilstrup, D.M., Burnett, J.C., Menke, K.K., Stensrud, S.L. \& Holmes, D.R. Jr. (1991) A randomized comparison of the nephrotoxicity of iopamidol and diatrizoate in high risk patients undergoing cardiac angiography. J. Am. Coll. Cardiol., 17, 384-390.

Worthley, L.I., Guerin, M. \& Pain, R.W. (1987) For calculating osmolality, the simplest formula is the best. Anaesth. Intensive Care, 15, 199-202. 